\title{
A Compact Low-Permittivity Dual-Layer EBG Structure for Mutual Coupling Reduction
}

\author{
A. Azarbar' ${ }^{1}$ and J. Ghalibafan ${ }^{2}$ \\ ${ }^{1}$ Islamic Azad University, Parand Branch, Tehran, Iran \\ ${ }^{2}$ Islamic Azad University, Dezful Branch, Dezful, Iran \\ Correspondence should be addressed to J. Ghalibafan, javad.ghalibafan@gmail.com
}

Received 15 February 2011; Revised 13 April 2011; Accepted 9 June 2011

Academic Editor: Hoi Shun Lui

Copyright (C) 2011 A. Azarbar and J. Ghalibafan. This is an open access article distributed under the Creative Commons Attribution License, which permits unrestricted use, distribution, and reproduction in any medium, provided the original work is properly cited.

\begin{abstract}
Electromagnetic bandgap (EBG) structures can help in the reduction of mutual coupling by their capabilities of suppressing surface wave's propagation in a specific frequency range. In this work, a dual-layer EBG structure, which had a lower resonant frequency than the single-layer one, is proposed in order to reduce the mutual coupling between $E$-plane coupled microstrip antenna array. As this EBG structure significantly made the series capacitance between neighbor cells larger, a drastic reduction of the unit cell size was achieved. The simulated and experimental results show that the proposed structure has a significant $19 \mathrm{~dB}$ mutual coupling reduction.
\end{abstract}

\section{Introduction}

Mutual coupling effects can cause significant degradation of performance in many array applications. For example, it may cause scan blindness in phased arrays with a large scanning angle and degradation of the side lobe level, especially for the ultra low side lobe level antenna arrays $[1,2]$.

In fact, two patches can be coupled in an array through two paths; the first path arises from the free space radiation which is present in all types of array antennas and the second path arises from surface waves constituting a very important factor in patch antennas. Generally, when a patch antenna operates in the fundamental mode, surface waves are strongly excited in the E-plane.

There are many methods for reducing the effects of mutual coupling, which include optimizing antenna dimensions [3], grooving the dielectric [4], covering the patch by additional dielectric layers [5], using shorting pins to cancel the capacitive polarization currents of the substrate [6], adding parasitic conducting tape to the middle of two antennas $[7,8]$, or using the dielectric as a bandgap structure between elements in the array $[9,10]$.

Electromagnetic bandgap structure consists of a periodic structure made of dielectric or metal and exhibits one or more forbidden frequency bands. Since the bandgap frequency range is determined by the physical dimension of the EBG and the optimum element separation for avoiding grating lobes in the visible region of the phase array antenna has to be $0.5 \lambda_{0}$ (where $\lambda_{0}$ is the free-space wavelength), reduction of mutual coupling by means of an EBG structure becomes particularly challenging when grating lobes must be avoided. In order to overcome this issue, many methods have been proposed to reduce dimensions of an EBG cell. They include using spiral EBG cells [11], loading lumped element in the EBG structure [12], adding multilayer dielectric substrate [13], and using Ferrite EBG structure [14].

In this work, a dual-layer EBG structure is proposed to reduce the mutual coupling of the probe-fed microstrip patch antenna arrays coupled in the E-plane which has a stronger surface wave than that of $H$-plane arrays. The proposed dual layer structure was inserted between antenna elements. In contrast to some other works [15], two lowpermittivity layers were used in this work and made the EBG unit cell size considerably smaller than the patch antenna size without sacrificing antenna performance. In addition, in the proposed EBG structure, the series capacitance between neighbor cells was enlarged in order to compact the cells. 


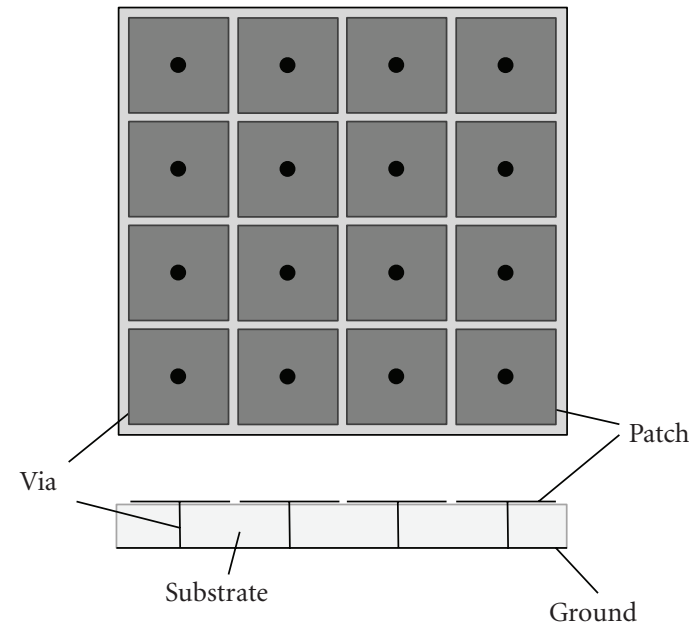

(a)

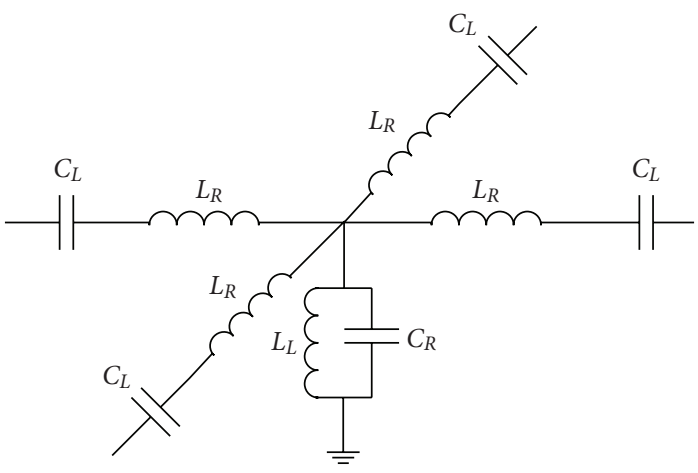

(b)

FIGURE 1: (a) Mushroom-like EBG structure, (b) equivalent circuit model of the conventional EBG unit cell [12].

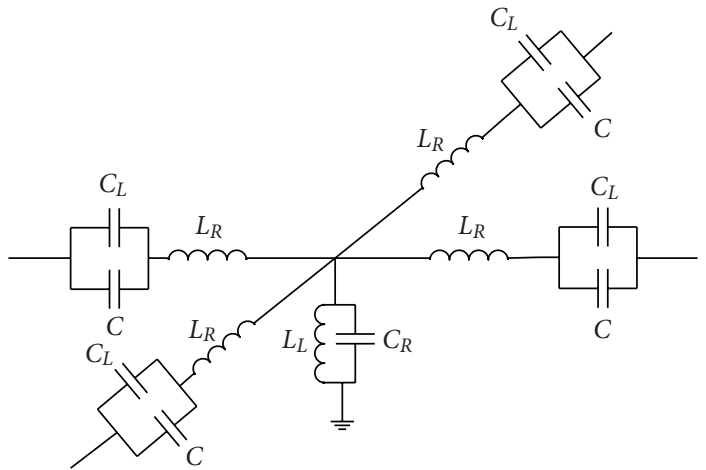

FIGURE 2: Equivalent circuit model of the proposed EBG unit cell.

In the first part, the design of the proposed unit cell is presented. In the second part, the effects of the EBG structure in reducing the mutual coupling of patch antenna arrays coupled in the E-plane are investigated, and the results are discussed later.

\section{EBG Structure Design}

2.1. Introducing of Conventional and Proposed EBG Unit Cell. The mushroom-like EBG structure was first proposed in [16]. It consisted of four parts: a ground plane, a dielectric substrate, metallic patches, and connecting vias. This EBG structure exhibited a distinct stopband for the surfacewave propagation and its operation mechanism could be explained by a combination of series LC circuits, and a parallel LC circuit as shown in Figure 1 [12-17]. In this case, the stop and start frequencies of the band gap were given by these equations [17]:

$$
f_{1}, f_{2}=\frac{1}{2 \pi \sqrt{L_{R} C_{L}}}, \frac{1}{2 \pi \sqrt{L_{L} C_{R}}},
$$

where the parameters $L_{L}, C_{L}, L_{R}$, and $C_{R}$ represent shunt inductance, series capacitance, series inductance, and shunt capacitance, respectively. From (1), it can be seen that in order to achieve an even more compact EBG structure, the capacitances and inductances should be increased, but in the EBG design procedure, if the dielectric material and its thickness are chosen, the inductances cannot be altered. Therefore, only the capacitances can be enlarged.

Here, to compact an EBG unit cell, the series capacitance was enlarged. In this case, the circuit model of conventional unit cell changed to a circuit model shown in Figure 2. In addition, (1) was replaced by

$$
f_{1}, f_{2}=\frac{1}{2 \pi \sqrt{L_{R}\left(C_{L}+C\right)}}, \frac{1}{2 \pi \sqrt{L_{L} C_{R}}} .
$$

As shown in Figure 2, capacitance $C$ was added in parallel to $C_{L}$. In this work, capacitance $C$ was achieved by adding a rectangular metallic ring to the layer under the EBG layer. Figure 3 shows the conventional and proposed EBG unit cells. 


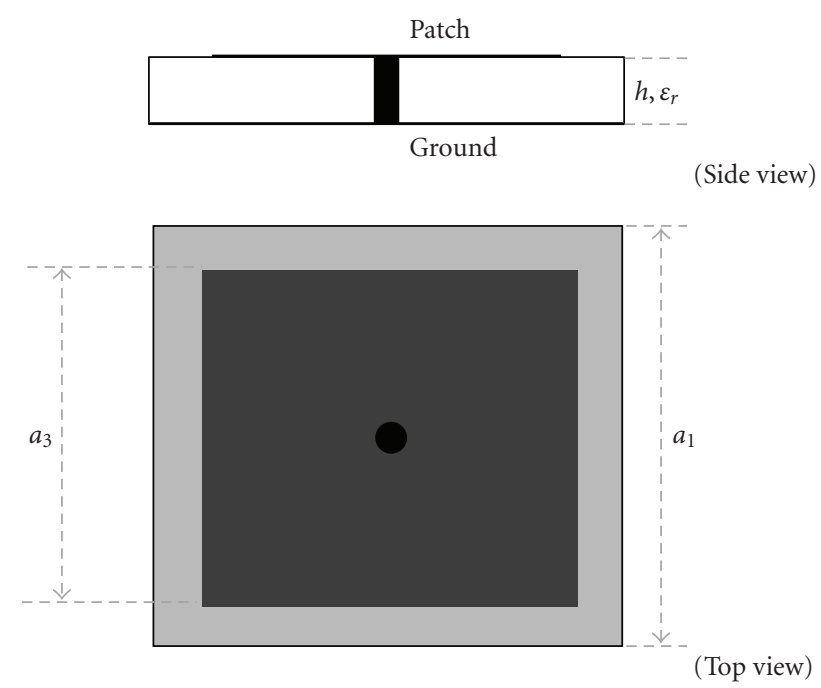

(a) Conventional EBG unit cells

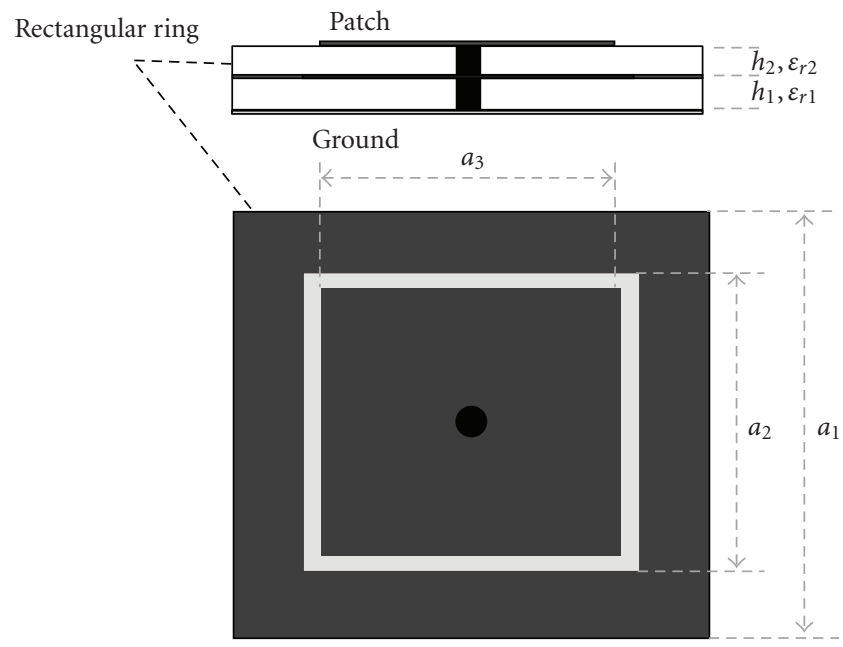

(b) Proposed EBG unit cells

FIgURE 3: Conventional and proposed EBG unit cells.

At bandgap, an EBG cell was characterized by a high value of surface impedance and a reflection phase of zero. As shown in Figure 4, the impedance surface was obtained by applying periodic boundary conditions on a cell and by setting the wave port impedance up to the top of the cell. Using the finite element solver HFSS, the impedance surface and reflection phase were computed under a normal incidence.

2.2. Compressing Conventional and Proposed EBG Unit Cells. Figure 5 shows the surface impedance for conventional and proposed EBG unit cells. In this case, both cells have similar dimensions. As shown in this figure, the proposed cell has a lower resonant frequency than the conventional one. This resonant frequency reduction is the result of series capacitance enlargement. When $a_{2}$ decreases, the width of rectangular ring increases; so, the series capacitance $(C)$ grows and the resonant frequency moves to lower frequencies (Figure 5).

\section{Antenna Design and the Results}

The E-plane coupled microstrip antenna arrays suffer from strong mutual coupling because of surface waves. Due to the capability of EBG structures to suppress surface waves, a single string of EBG cells was inserted between antenna elements in order to reduce the mutual coupling (Figure 6). Because a single string of EBG cells was inserted between the elements, the EBG cells in Figure 6 are one-dimensional (they were repeated only in the $x$ direction), while the EBG structures in Figure 3 are two-dimensional (they were repeated in $x$ and $y$ directions). In this case, to increase the series capacitance between the cells, the rectangular ring in Figure 3 was replaced with the metallic ribbon in Figure 6.

Most proposals found in the literature have used thin, high-permittivity substrates for reducing the size of EBG cells, and both the periodic structure and patch antenna have been printed on the same layer; however, the highpermittivity layer has decreased the bandwidth and directivity of the antenna. In this work, the low-permittivity layers were used for increasing the bandwidth and directivity of antenna. Reduction of the permittivity layers will increase the size of the EBG cell. To reduce the size of the EBG cell, the series capacitance $(C)$ was increased.

HFSS numerical simulation was used to simulate the $E$ plane coupled microstrip antennas on a dielectric substrate with $h_{1}=0.508 \mathrm{~mm}, h_{2}=0.381 \mathrm{~mm}, \varepsilon_{r 1}=3.55$, and $\varepsilon_{r 2}=2.2$. To obtain the resonant frequency at $5 \mathrm{GHz}$, the rectangular patch's size was $10 \mathrm{~mm} \times 17.9 \mathrm{~mm}$, and to avoid the grating lobe, the distance between the patches was $30 \mathrm{~mm}$ $(0.5 \lambda)$.

The optimum values of the structural parameters of the antenna are as follows.

The simulated mutual coupling $\left(S_{12}\right)$ and reflection coefficient $\left(S_{11}\right)$ in both cases (i.e., with and without EBG) are compared in Figure 7. As shown in this figure, the proposed configuration improves the mutual coupling between patch elements.

The proposed antenna with optimum dimensions was fabricated. Figure 8 shows the measured mutual coupling and reflection coefficient. In this figure, the simulated results are in a reasonable agreement with the measured results. As a consequence, for the case of a single string of the proposed EBG, a $19 \mathrm{~dB}$ reduction in the mutual coupling was achieved in the array with EBG, which is not the case for the array without the EBG.

\section{Conclusions}

In this paper, a novel compact EBG structure was studied for the array miniaturization with the reduced mutual coupling. In such a structure, the series capacitance was enlarged 


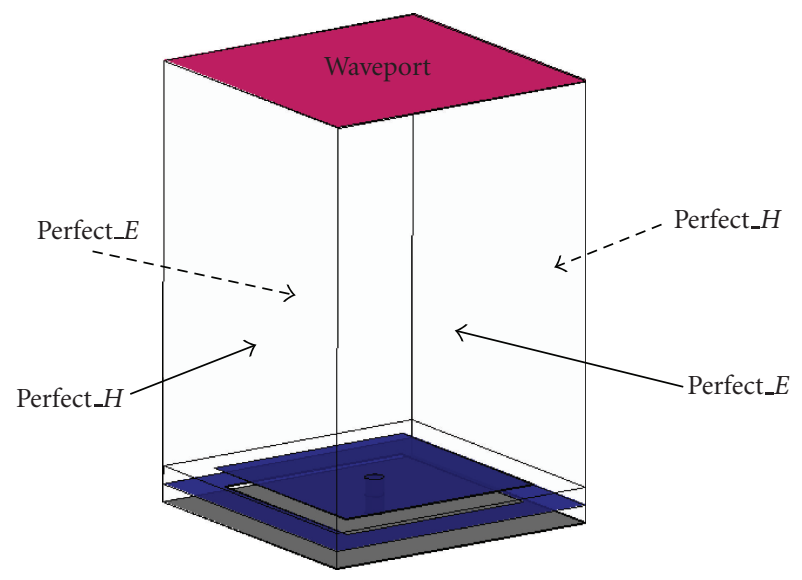

FIGURE 4: EBG cell simulated with periodic boundary conditions under a normal incident wave.

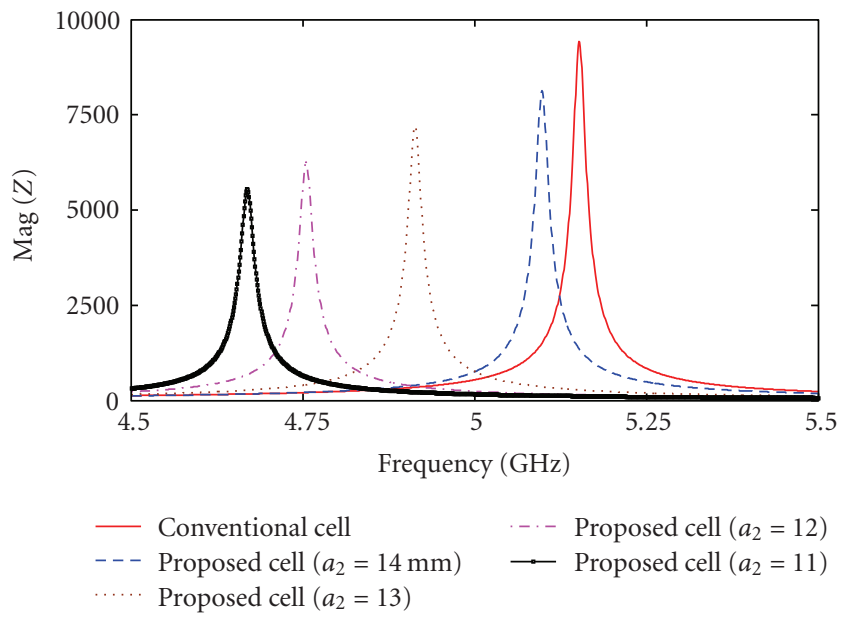

Figure 5: Simulated surface impedance of conventional and proposed EBG cells. $a_{1}=16 \mathrm{~mm}, a_{3}=14 \mathrm{~mm}, R_{\text {via }}=0.5 \mathrm{~mm}, h_{1}=h_{2}=$ $0.508 \mathrm{~mm}, h=h_{1}+h_{2}, \varepsilon_{r}=\varepsilon_{r 1}=\varepsilon_{r 2}=3.55$.

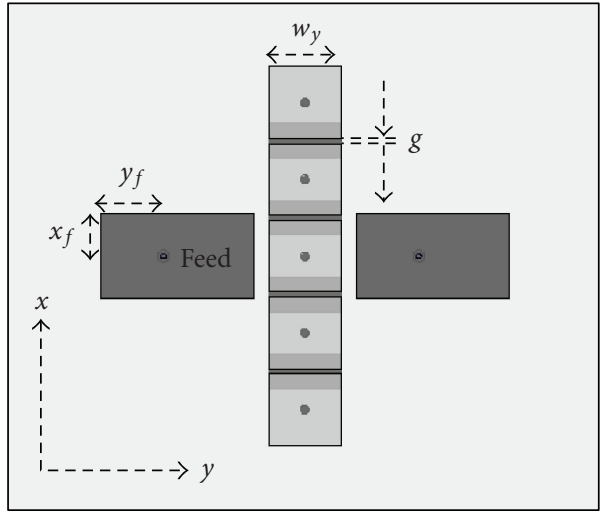

(a) Upper layer

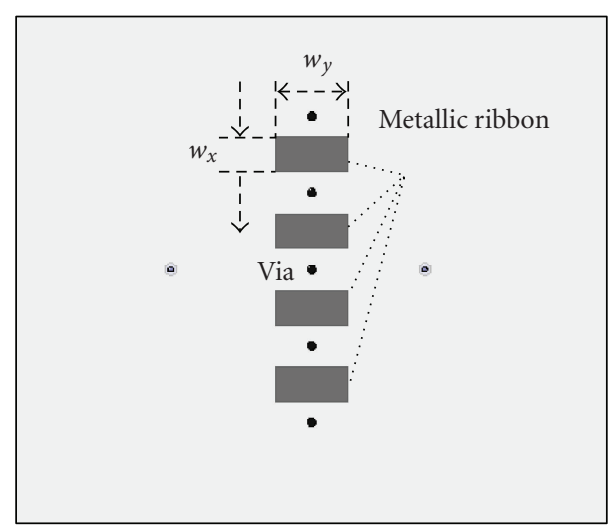

(b) Bottom layer

FIGURE 6: Geometry of an E-plane coupled microstrip antenna array with the proposed EBG structure. $w_{y}=8.4 \mathrm{~mm}, w_{x}=4.05 \mathrm{~mm}$, $R_{\text {via }}=0.5 \mathrm{~mm}, g=0.6 \mathrm{~mm}, x_{f}=5 \mathrm{~mm}, y_{f}=8 \mathrm{~mm}$. 


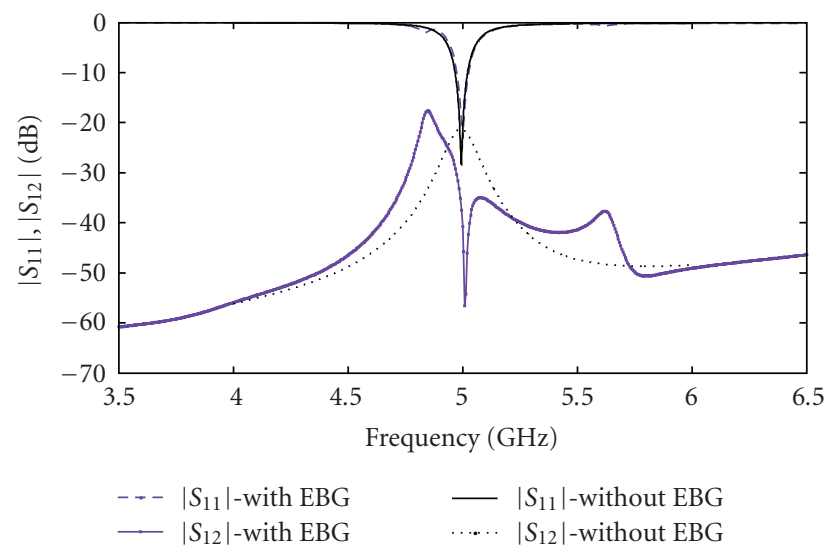

Figure 7: Simulated mutual coupling and reflection coefficient of the E-plane coupled microstrip antenna array with and without EBG.

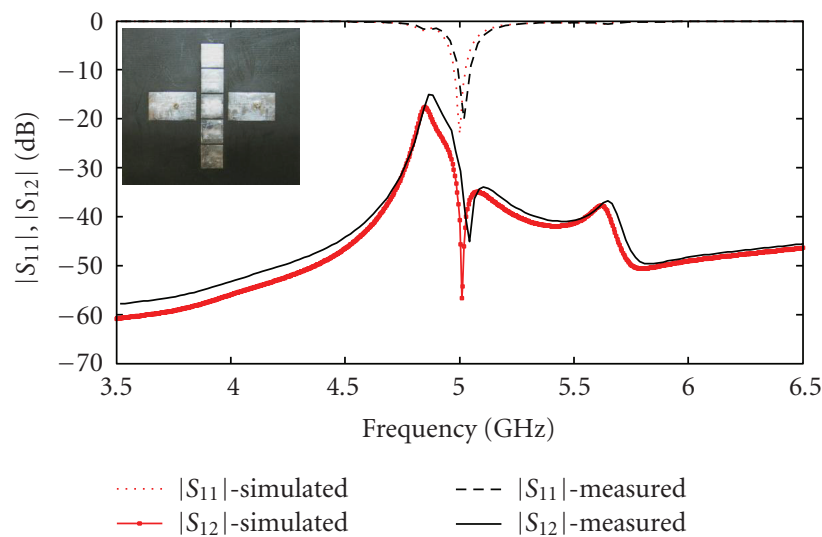

FIGURE 8: Simulated and measured mutual coupling and reflection coefficient of E-plane, coupled microstrip antenna array with EBG.

between the neighbor cells by adding a rectangular metallic ring to a layer under the EBG layer. The EBG structure was analyzed using the HFSS numerical simulation, and it was compared with the conventional EBG structure. A single string of the EBG structure was inserted between antenna elements in an $E$-plane coupled microstrip antenna array in order to reduce the mutual coupling. Compared with the case without the EBG, the case of a single string of the proposed EBG achieved a $19 \mathrm{~dB}$ reduction in mutual coupling. This mutual coupling reduction technique can be used in various antenna array applications.

\section{References}

[1] D. M. Pozar and D. H. Schaubert, "Analysis of an infinite array of rectangular microstrip patches with idealized probe feeds," IEEE Transactions on Antennas and Propagation, vol. 32, no. 10, pp. 1101-1107, 1984.

[2] D. M. Pozar and D. H. Schaubert, "Scan blindness in infinite phase arrays of printed dipoles," IEEE Transactions on Antennas and Propagation, vol. 32, no. 6, pp. 602-610, 1984.

[3] M. A. Khayat, J. T. Williams, D. R. Jackson, and S. A. Long, "Mutual coupling between reduced surface-wave microstrip antennas," IEEE Transactions on Antennas and Propagation, vol. 48, no. 10, pp. 1581-1593, 2000.
[4] G. Kumar and K. P. Ray, Broadband Microstrip Antennas, Artech House, Norwood, Mass, USA, 1996.

[5] N. G. Alexopoulos and D. R. Jackson, "Fundamental superstrate (cover) effects on printed circuit antennas," IEEE Transactions on Antennas and Propagation, vol. 32, no. 8, pp. 807816, 1984

[6] M. M. Nikolić, A. R. Djordjević, and A. Nehorai, "Microstrip antennas with suppressed radiation in horizontal directions and reduced coupling," IEEE Transactions on Antennas and Propagation, vol. 53, no. 11, pp. 3469-3476, 2005.

[7] H. Xin, K. Matsugatani, M. Kim et al., "Mutual coupling reduction of low-profile monopole antennas on highimpedance ground plane," Electronics Letters, vol. 38, no. 16, pp. 849-850, 2002.

[8] K. S. Min, D. J. Kim, and Y. M. Moon, "Improved MIMO antenna by mutual coupling suppression between elements," in Proceedings of the 8th European Conference on Wireless Technology, pp. 125-128, October 2005.

[9] S. D. Cheng, R. Biswas, E. Ozbay, S. McCalmont, G. Tuttle, and K.-M. Ho, "Optimized dipole antennas on photonic band gap crystals," Applied Physics Letters, vol. 67, no. 23, pp. 3399-3401, 1995.

[10] Y. Q. Fu, Q. R. Zheng, Q. Gao, and G. H. Zhang, "Mutual coupling reduction between large antenna arrays using electromagnetic bandgap (EBG) structures," Journal of Electromagnetic Waves and Applications, vol. 20, no. 6, pp. 819-825, 2006. 
[11] Q.-R. Zheng, B.-Q. Lin, Y.-Q. Fu, and N.-C. Yuan, “Characteristics and applications of a novel compact spiral electromagnetic band-gap (EBG) structure," Journal of Electromagnetic Waves and Applications, vol. 21, no. 2, pp. 199-213, 2007.

[12] T. Yanagi, T. Oshima, H. Oh-hashi et al., "Lumped-element loaded EBG structure with an enhanced bandgap and homogeneity," in Proceedings of the IEEE International Workshop on Antenna Technology: Small Antennas and Novel Metamaterials (IWAT '08), pp. 458-461, Chiba, Japan, March 2008.

[13] S. M. Moghadasi, A. R. Attari, and M. M. Mirsalehi, "Design of three-layer circular mushroom-like EBG structures," in Proceedings of the Electromagnetics Research Symposium, pp. 143-146, Hangzhou, China, 2008.

[14] B. Gao and M. F. Yuen, "Passive UHF RFID with ferrite Electromagnetic Band Gap (EBG) material n for metal objects tracking," in Proceedings of 58th Electronic Components and Technology Conference(ECTC '08), pp. 1990-1994, Lake Buena Vista, Fla, USA, May 2008.

[15] H. S. Farahani, M. Veysi, M. Kamyab, and A. Tadjalli, "Mutual coupling reduction in patch antenna arrays using a UC-EBG superstrate," IEEE Antennas and Wireless Propagation Letters, vol. 9, pp. 57-59, 2010.

[16] D. Sievenpiper, L. Zhang, R. F. Jimenez Broas, N. G. Alexöpolous, and E. Yablonovitch, "High-impedance electromagnetic surfaces with a forbidden frequency band," IEEE Transactions on Microwave Theory and Techniques, vol. 47, no. 11, pp. 2059-2074, 1999.

[17] A. Sanada, C. Caloz, and T. Itoh, "Characteristics of the composite right/left-handed transmission lines," IEEE Microwave and Wireless Components Letters, vol. 14, no. 2, pp. 68-70, 2004. 

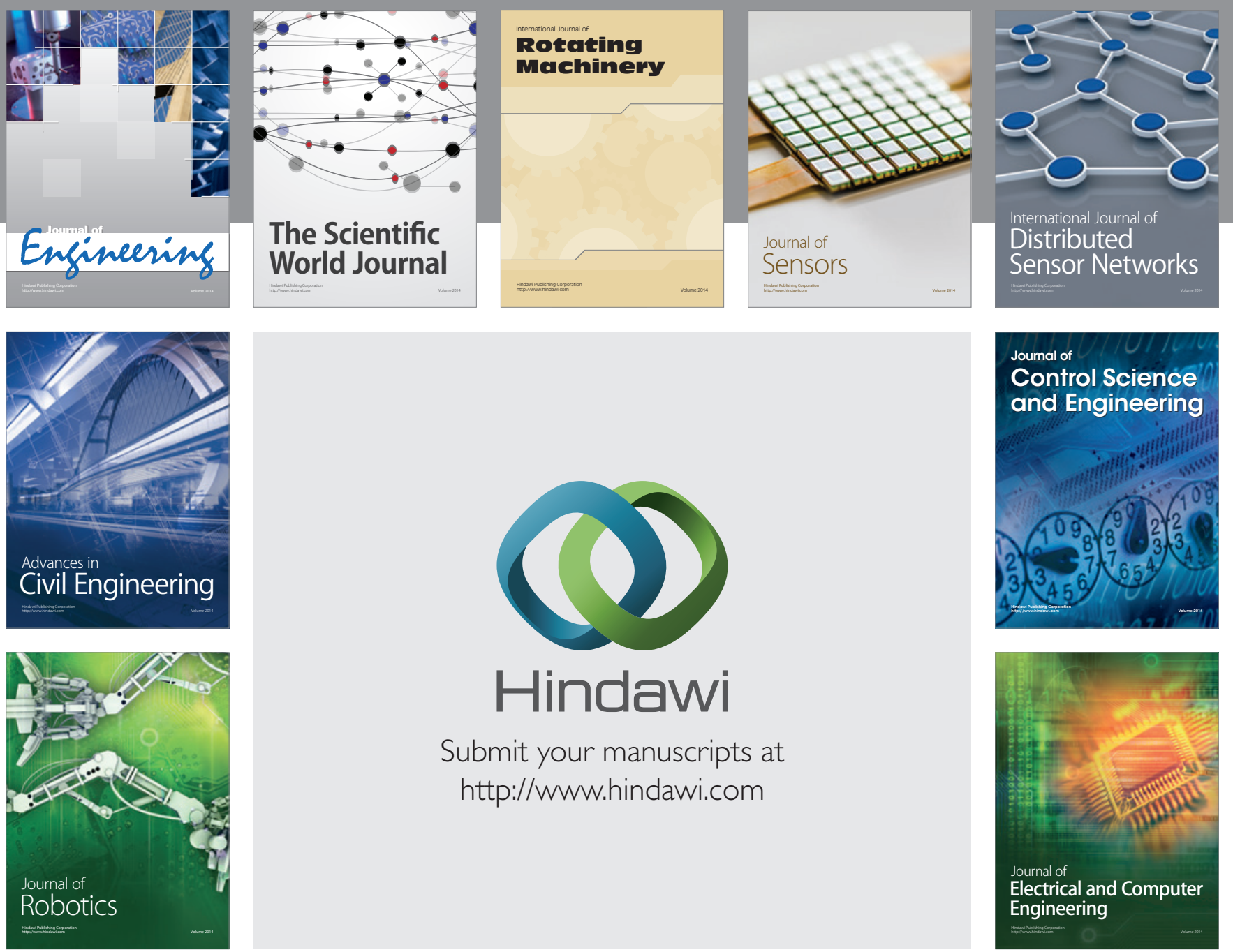

Submit your manuscripts at

http://www.hindawi.com
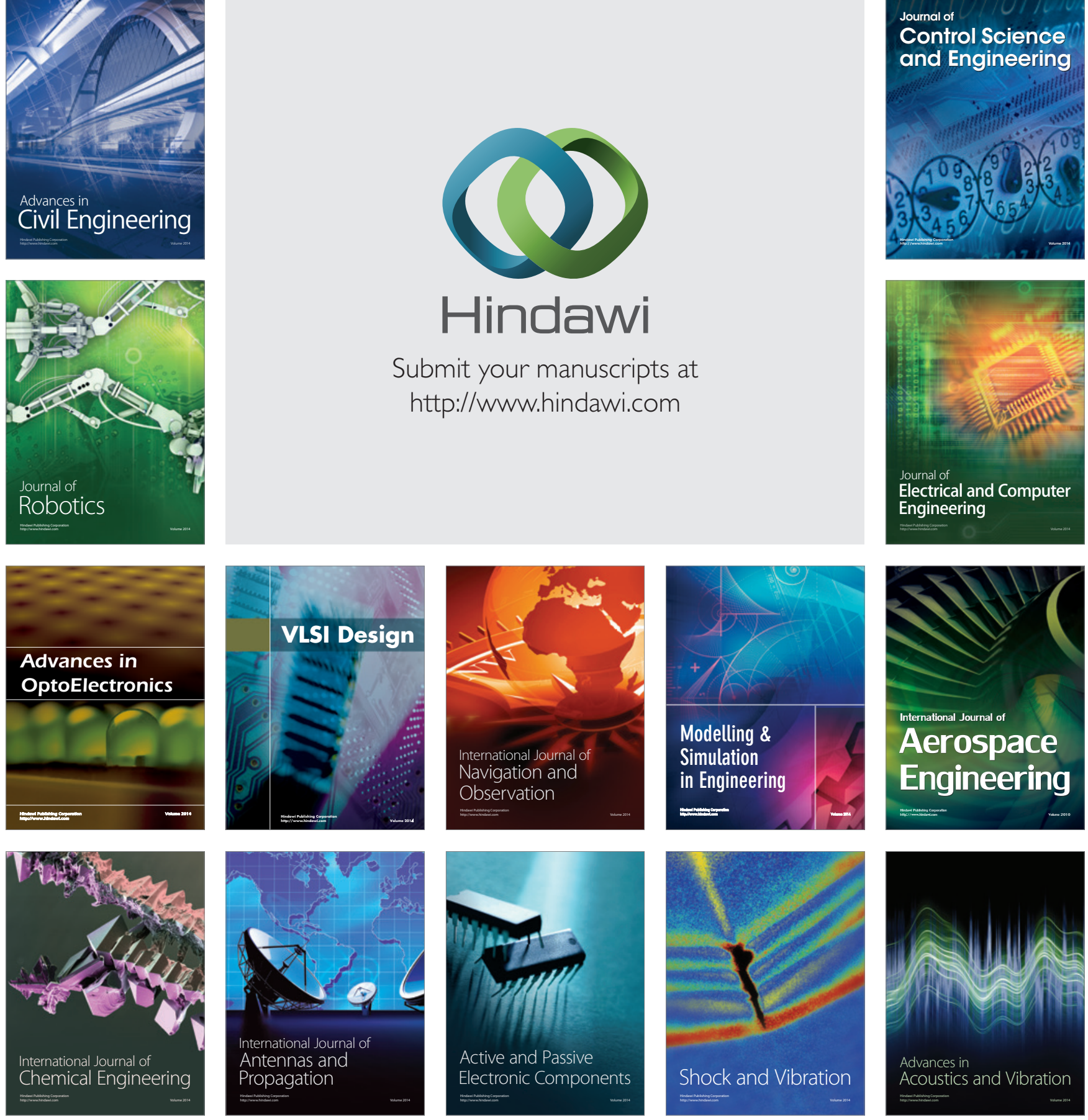